Ventricular haemodynamic parameters were also measured, include HR, LVSP. Left ventricular myocardial was separated and cut to five slice. After experiment, the myocardial was used for myocardial infarction size evaluated with TTC stained. Immunohistochemical staining for Phosphorylation Akt and GSK-3 $\beta$ expression.

Results Ischemic postconditioning reduced $\mathrm{LDH}, \mathrm{CK}$ and improved the haemodynamic parameters, and reduced myocardial infarction size (29.5\% vs $47.3 \%$ ). phospho-Akt and phospho-GSK-3 $\beta$ expression increased markedly in IPost group. Wortmannin may reduced phospho-Akt expression, and phospho-GSK-3 $\beta$ expression increased in $\mathrm{I} / \mathrm{R}+\mathrm{SB}$ group.

Conclusion Ischemic postconditioning may synergically protect myocardium in isolated rat heart. Wortmannin, a inhibitor of Akt, may weaken the cardioprotection effect of postconditioning. SB216763, as a inhibitor of GSK-3 $\beta$, can simulate cardioprotection effect of postconditioning. Akt and GSK-3 $\beta$ play important role in the mechanism of signal pathway in ischaemia postconditioning.

\section{e0120 THE IMPACT OF DIABETES ON THE ROLE OF REPERFUSION INJURY SALVAGE KINASE PATHWAY}

doi:10.1136/hrt.2010.208967.120

Zhao Xin, Yu Xuefan, Quan Nanhu. Department of Cardiology, First Hospital of Jilin University, ChangChun, China

Objective To elucidate the effects of postconditioning on ischaemia/ reperfusion cardiac and the role of reperfusion injury salvage kinase pathway in type 2 diabetic rats.

Methods The type 2 diabetic rats were induced by the intravenous injection of streptozotocin and high caloric diet. 60 Wister rats were divided into three groups randomly. Ischaemia- reperfusion in nomal rats (A group), ischaemia postconditioning in nomal rats (B group), ischaemia postconditioning in diabetic rats (C group). Rats were used for Langendorff isolated heart perfusion with $30 \mathrm{~min}$ of globe ischaemia and 60 min of reperfusion, then the models of Ischaemiareperfusion (A) were made. But to B and $\mathrm{C}$, rat hearts were subjected to six cycles of $10 \mathrm{~min}$ of globe ischaemia and $10 \mathrm{~min}$ of reperfusion as ischaemia postconditioning during the early minutes of reperfusion. Phosphorylation of akt and gsk-3 $\beta$ were analysed by western blotting and immunohistochemical staining.

Results phospho-akt and phospho-gsk-3 $\beta$ expression increased markedly in B group. But compared A group there were no parently diffrence in $\mathrm{C}$ group. phospho-akt and phospho-gsk- $3 \beta$ expression in $\mathrm{C}$ group is more less than in $\mathrm{B}$ group.

Conclusion Ischemic postconditioning may significiently protect myocardium in isolated nomal rat hearts. But in diabetic rats the protection of Ischaemic postconditioning has no effect, the mechanism of this phenomina maybe connected with gsk-3 $\beta$ in the condition of diabetic.

\section{e0121 THE EFFECTS OF ENDOTHEIN-1 AND BQ-123 ON ATPASE ACTIVITY AND MRNA EXPRESSION IN AORTIC SMOOTH MUSCLE CELLS FROM SPONTANOUSLY HYPERTENSIVE RATS}

doi:10.1136/hrt.2010.208967.121

${ }^{1}$ Qian-Hui Shang, 'Zheng Yang, ${ }^{1}$ Qian-feng Jiang, ${ }^{2} Y u$ Xiao. ${ }^{1}$ Institute of Clinical Medine of Zunyi Medical College, Department of Cardiology, Affiliated Hospital of Zunyi Medical College, Guizhou, China; ${ }^{2}$ Key Laboratory of Cell Engineering of Guizhou Province, Zunyi, Guizhou, China

Aim To study the effects of endothelin-1 (ET-1) and BO-123 $\left(\mathrm{ET}_{\mathrm{A}}\right.$ receptor antagonist) on activities and mRNA expression of ATPase in aortic smooth muscle cells (ASMCs) from spontaneously hypertensive rats (SHR) and Wistar-Kyoto (WKY) rats.
Methods The ASMCs were isolated from SHR and WKY rats. The ATPase activities of cultured ASMCs were determined by spectrophotography. The mRNA levels of $\mathrm{Na}^{+}, \mathrm{K}^{+}$-ATPase $\alpha_{1}$-subunit and plasma membrane $\mathrm{Ca}^{2+}$-ATPase isoform $1\left(\mathrm{PMCA}_{1}\right)$ were measured by semiquantitative reverse transcription PCR (RT-PCR). Results 3 different concentrations of ET-1 $\left(1 \times 10^{-9}, 1 \times 10^{-8}\right.$ and $1 \times 10^{-7} \mathrm{~mol} / \mathrm{l}$ ) significantly attenuated the activities of $\mathrm{Na}^{+}, \mathrm{K}^{+}$ATPase and $\mathrm{Ca}^{2+}$-ATPase and $\mathrm{PMCA}_{1}$ mRNA expression (all $p<0.01$ ) in ASMCs from SHR. Three different concentrations of $\mathrm{BQ}-123\left(1 \times 10^{-8}, 1 \times 10^{-7}\right.$ and $\left.1 \times 10^{-6} \mathrm{~mol} / \mathrm{l}\right)$ obviously prevented ET-1 mediated the inhibition of two kinds ATPase activities (all $\mathrm{p}<0.01$ ) and downregulation of $\mathrm{PMCA}_{1}$ mRNA expression $(p<0.01)$. But the mRNA expression level of $\mathrm{Na}^{+}, \mathrm{K}^{+}$-ATPase $\alpha_{1}$ subunit had no alteration after intervened by ET-1 ( $p>0.05)$.

Conclusions ET-1 may suppress $\mathrm{Na}^{+}, \mathrm{K}^{+}$-ATPase, $\mathrm{Ca}^{2+}$-ATPase activities via $\mathrm{ET}_{\mathrm{A}}$ receptor. The influence of $\mathrm{ET}-1$ on $\mathrm{Ca}^{2+}$-ATPase activity may partially occur in the transcriptional level. BQ-123 can inhibit the effect of ET-1 on two kinds ATPase activities of ASMCs in SHR by blocking the $\mathrm{ET}_{\mathrm{A}}$ receptor.

\section{e0122 EFFECTS OF CARDIOTROPHIN-1 C-TERMINAL PEPTIDES ON CARDIOMYOCYTE APOPTOSIS IN SD RATS FOLLOWING MYOCARDIAL ISCHAEMIA REPERFUSION INJURY}

doi:10.1136/hrt.2010.208967.122

Zhen Yao, Shufen Chen, Yunbo Zhang, Wei Zhang, Xiaofang Zhang, Shijiao Yan. The People's Hospital of Sanya

Objective Observation of CT-1 C-terminal peptides in ischaemia -reperfusion injury before and after the intervention on myocardial cell apoptosis in SD rats.

Methods With ligation-release SD rats left posterior descending branch of coronary artery the ischaemia reperfusion heart model was established. $27 \mathrm{SD}$ rats were randomly divided into four groups: Normal group $(\mathrm{N}, \mathrm{n}=5)$; Disease group $(\mathrm{D}, \mathrm{n}=6)$, Beginning of reperfusion after $30 \mathrm{~min}$ of $\mathrm{MI}$; MI/R post-intervention group ( $\mathrm{T}$, $\mathrm{n}=8)$, Intraperitoneal injection of CT-1 C-terminal peptide $(100 \mu \mathrm{g} /$ $\mathrm{kg}$ ) at same time of beginning of reperfusion after $30 \mathrm{~min}$ of $\mathrm{MI}$; $\mathrm{MI} / \mathrm{R}$ pre-intervention group $(\mathrm{O}, \mathrm{n}=8), \mathrm{MI} / \mathrm{R}$ experiments was performed after intraperitoneal injection of CT-1C-terminal peptide $(100 \mu \mathrm{g} / \mathrm{kg})$ for 7 days. In accordance with the ECG monitoring results ended the experiment in animals dying, left the serum for examination of concentrations of $\mathrm{CK}$ and $\mathrm{MDA}$ and cut the ischaemic heart tissue and surrounding areas fixed in neutral solution of formaldehyde, paraffin-embedded and sliced. Using endlabelling TUNEL assay apoptosis of myocardial cells and calculate the cardiac myocyte apoptotic index (AI).

Results After MI/R, the average survival time of the disease group of $\mathrm{SD}$ rats was $93.17 \pm 24.7 \mathrm{~min}$, that of $\mathrm{MI} / \mathrm{R}$ pre-intervention group was $87.88 \pm 18.3 \mathrm{~min}$. The average survival time of $\mathrm{MI} / \mathrm{R}$ postintervention group was $155.5 \pm 80.13 \mathrm{~min}$, significantly longer than that of the disease and $\mathrm{MI} / \mathrm{R}$ pre-(chronic) intervention group $(\mathrm{p}<0.01)$; The serum CK activity and MDA content and the myocardial apoptotic index (AI) around infarct area were increased significantly in disease group ( $\mathrm{N}$ vs $\mathrm{D}, \mathrm{p}<0.01$ ), and which has been reduced significantly in the post-intervention group ( $T$ vs $D$, $\mathrm{p}<0.01$ ). But still higher than that of normal group ( $q$ values were 5.197, 5.782, 7.391, respectively; $p<0.01$ ); The serum CK activity and MDA content were more higher in pre-ischaemia group than that in the disease group, and their apoptosis index (AI) is higher than that of normal group $(p<0.01)$, but was no significant difference compared with the disease group $(p>0.05)$; There were significant correlations between the myocardial apoptosis with myocardial injury and the extent of oxidative damage ( $r$ values were $0.9245,0.8679$, respectively; $\mathrm{p}<0.01$ ). 
Conclusion Short-term use of CT-1C-terminal peptide early in reperfusion can reduce myocardial tissue injury and oxidative damage, as well as the extent of cardiomyocyte apoptosis, so that the extension of animal survival time; but the intraperitoneal injection of CT-1C-terminal peptide after a longer period of time reduced the tolerance of SD rats on ischaemia reperfusion injury, the tissue injury and the extent of oxidative damage increased significantly, and cardiac myocyte apoptosis have occurred in the surrounding area of infarction, and the animals have a shorter survival time.

\section{e0123 THE EFFECT OF DIABETES ON PROTECTION OF ISCHAEMIC POSTCONDITIONING IN MYOCARDIAL ISCHAEMIA- REPERFUSION INJURY}

doi:10.1136/hrt.2010.208967.123

Zhao Xin, Yu Xuefan, Quan Nanhu. Department of Cardiology, First Hospital of Jilin University, Changchun, China

Objective Study on the effect of diabetes on protection of ischaemia Postconditioning in myocardial ischaemia-reperfusion injury in isolated rat hearts.

Methods The type 2 diabetic rats were induced by the intravenous injection of streptozotocin (STZ) and high caloric diet. 60 Wister rats were divided into three groups randomly. Ischaemia- reperfusion in nomal rats (A group), ischaemia postconditioning in nomal rats (B group), ischaemia postconditioning in diabetic rats ( $\mathrm{C}$ group). Rats were used for Langendorff isolated heart perfusion with $30 \mathrm{~min}$ of globe ischaemia and $60 \mathrm{~min}$ of reperfusion, then the models of Ischaemia- reperfusion (A) were made. But to B and C, rat hearts were subjected to six cycles of $10 \mathrm{~s}$ of globe ischaemia and $10 \mathrm{~s}$ of reperfusion as ischaemia postconditioning during the early minutes of reperfusion. The levels of lactate dehydrogenase $(\mathrm{LDH})$ in the coronary effluent and infarction size was determined by TTC staining. Phosphorylation of akt and gsk- $3 \beta$ were analysed by western blotting and immunohistochemical staining.

Results Ischemic postconditioning reduced $\mathrm{LDH}, \mathrm{CK}$ and improved the haemodynamic parameters and reduced myocardial infarction size $(29.50 \pm 3.4 \%$ vs $45.65 \pm 4.8 \%$ ), phospho-Akt and phospho-GSK$3 \beta$ expression increased markedly in B group. But compared A group there were no parently diffrence in $\mathrm{C}$ group. The level of $\mathrm{LDH}, \mathrm{CK}$ didn't decline and the myocardial infarction size were not reduced. phospho-Akt and phospho-GSK-3 $\beta$ expression in C group is more less than in B group.

Conclusion Ischemic postconditioning may significantly protect myocardium from reperfusion injury in isolated normal rat hearts. But in diabetic rats, the protection of Ischaemic postconditioning has no effect, the mechanism of this phenomenon maybe connected with lower expression of Phosphorylation of Akt and GSK-3 $\beta$ in the condition of diabetic and impaired Reperfusion Injury Salvage Kinase (RISK) signalling pathway (RISK pathway).

\section{e0124 EFFECTS OF OXIDATIVE STRESS AND GENDER DIFFERENCES IN SD RATS WITH HIGH-SALT HYPERTENSION VIA ACUTE SHORT-TERM COLD EXPOSURE}

doi:10.1136/hrt.2010.208967.124

Lei Guangtao, Wu Qinghua. Second Affiliated Hospital of Nanchang University, Nanchang, China

Objective To perform high-salt hypertension model in SD rats and observe effects and oxidative stress and gender differences in SD rats, and then evaluate mechanism that blood pressure is affected by oxidative stress induced by cold environment.

Methods The male and female SD rats were randomly divided into four groups $(n=8)$ : male control group (MC), female control group
(FC), male high-salt group (MS), female high-salt group (FS), MC and FC were fed regularly, MS and FS were fed with diet composed of $8 \%$ salt. Feeding period was 8 weeks. Four groups were fed regularly in ninth week; in the first 10 weeks, four groups were put into a $4^{\circ} \mathrm{C}$ artificial climate box in tenth, $1 \mathrm{~h}$ per day. Systolic blood pressure (SBP) in SD rats was measured every other day from 1st week to in first 4 days 9 th week with tail cuff. Systolic blood pressure in SD rats was measured daily with tail cuff in late 3 days of 9 th week and 10th weeks.24-h urine in each group was collected by biological metabolism, calculated accurately.

Results 1. High-salt diet for 8 weeks, MS group and FS group blood pressure was significantly higher than the control group the same sex $(p<0.05)$. In 10th weekend four sets of blood pressure after cold exposure (BP) were higher, MS group and the FS group blood pressure $\triangle \mathrm{BP}(\triangle \mathrm{BP}=$ before exposure $\mathrm{BP}$-after exposure $\mathrm{BP})$ significantly higher than the control group the same sex $(p<0.01)$. 2. 8 week FS and MS $24 \mathrm{~h}$ urine volume, urinary MALB, urinary RBP, urine sodium, urine potassium excretion higher than that of the same sex control group $(p<0.01)$; MS and FS groups showed no change in exposure;3. After cold exposure high salt group $24 \mathrm{~h}$ urinary 8-isoPGF2 $\alpha$ excretion compared with before the cold exposure was significantly higher $(p<0.01)$, serum Ang II levels than before the cold increased and serum NO concentration decreased $(p<0.05)$, while no change in the control group. Before and after cold exposure the MS and FS, MC compared with FC no gender differences emerged. 4. After cold exposure $\mathrm{NADPH}$ oxidase activity and SOD activity, MS compared with FS, MC compared with FC does not appear gender differences, but the gender of the high salt group was significantly higher $(\mathrm{p}<0.05)$.

Conclusion 1. High-salt diet increased blood pressure, and high-salt diet on blood pressure after high salt gender differences emerged; resume normal diet of high salt hypertensive rats have a certain recovery of renal function, blood pressure, but high salt blood pressure, gender differences still exist in blood pressure. Control group with the same sex, short-term acute cold exposure for highsalt hypertensive rats blood pressure increased significantly; 2 . Cold high-salt hypertensive rats after exposure, oxidative stress increased; but male and female rats after exposure to cold and oxidative stress between the gender differences are not shown.

\section{e0125 MIMP-9 GENE POLYMORPHISMS CONTRIBUTE TO CORONARY ARTERY DISEASE RISK IN THE UIGHUR POPULATION OF CHINA}

doi:10.1136/hrt.2010.208967.125

Wang Lei, Ma Yitong, Yang Yining, Xie Xiang, Liu Fen. Department of Cardiovascular Medicine, The First Affiliated Hospital, Xinjiang Medical University, Urumqi, China

Background Matrix metalloproteinase-9 (MMP-9) plays a pivotal role in early atherosclerosis, vascular remodelling and development of atherosclerotic lesion. The potentially functional MMP-9 polymorphisms may contribute to the susceptibility of coronary artery disease (CAD). We aimed to investigate the association between three SNPs (-1562C > T, R279Q, R668Q) of the MMP-9 gene with $\mathrm{CAD}$ in the Uighur population of China.

Materials and methods 375 angiographic ally proven patients with coronary artery disease and 417 sex-matched and ethnically matched controls were genotyped for MMP-9 polymorphisms by the PCR-restriction fragment length polymorphism (PCR-RFLP) technique. Genotype/allele frequencies were compared in patients and controls using the $\chi^{2}$ test. The relationship between the polymorphism of the MMP-9 gene and the severity of coronary arterial stenosis was analysed also.

Results At MMP-9 -1562 locus, there were significant differences between patients and controls $(p<0.05)$, leading to significant $O R$ for TT genotype ( $\mathrm{OR}=2.93$, CI 1.03 to 8.72$)$ and $\mathrm{R}$ allele $(\mathrm{OR}=1.85$, 